\title{
LA RELACIÓN CHINA-ALEMANIA (2005-2018): ASOCIACIÓN ESTRATÉGICA Y PRAGMATISMO ECONÓMICO
}

\author{
THE CHINA-GERMANY RELATIONSHIP \\ (2005-2018): STRATEGIC PARTNERSHIP \\ AND ECONOMIC PRAGMATISM
}
LES RELATIONS CHINE-ALLEMAGNE (2005-2018):
L'ASSOCIATION STRATÉGIQUE, LE PRAGMATISME ÉCONOMIQUE

\author{
Manuel de Jesús Rocha Pino ${ }^{1}$ \\ Universidad Nacional Autónoma de México \\ rochapino@yahoo.com
}

Resumen: En este texto se analiza la trayectoria de la relación China-Alemania durante el periodo 2005-2018 y el contenido de la agenda bilateral que ambos países han impulsado. En la investigación se identifican dos prioridades compartidas: 1) la preferencia por el incremento de sus relaciones económicas y comerciales mediante el pragmatismo económico (aunque en detrimento de un diálogo sobre los valores de la buena gobernanza) y 2) el proceso de institucionalización de la agenda de cooperación bilateral por medio de la asociación estratégica integral China-Alemania. Se considera que el incremento en las relaciones de interdependencia económica es un incentivo fundamental para la ampliación de la agenda de cooperación en su asociación estratégica. De igual modo, la asociación estratégica es un espacio institucional para el acomodo de sus intereses y la diversidad de sus preferencias (por ej., el compromiso de Alemania con las relaciones transatlánticas o los principios estructurales de la política exterior de China, como el respeto a su soberanía).

Palabras clave: asociación estratégica integral China-Alemania; interdependencia; Unión Europea; institucionalismo liberal; asimetrías.

${ }^{1}$ La investigación fue elaborada gracias a una beca del Programa de Becas Posdoctorales en la unAm con la asesoría de la Dra. Yleana Cid Capetillo del Centro de Relaciones Internacionales de la Facultad de Ciencias Políticas y Sociales. Todos los errores y omisiones corresponden al autor. 
ABSTRACT: This paper analyzes the trajectory of China-Germany relations during the 2005-2018 period and the content of the bilateral agenda that both countries have promoted. The research identifies two priorities shared by the actors: 1) the preference for increasing their economic and commercial relations through economic pragmatism (although to the detriment of a dialogue on the values of good governance); 2) the process of institutionalization of the bilateral cooperation agenda through the China-Germany comprehensive strategic partnership. The research considers that the growth in their economic interdependence is a fundamental incentive for the expansion of the cooperation agenda into the strategic partnership. Likewise, the strategic partnership is an institution for the accommodation of their interests and the diversity of their preferences (i.e. Germany's commitment to transatlantic relations or the principles of China's foreign policy as respect for its sovereignty).

Keywords: China-Germany comprehensive strategic partnership; interdependence; European Union; liberal institutionalism; asymmetries.

\section{Traducción de Gonzalo Celorio Morayta}

Résumé: Ce texte analyse l'évolution des relations Chine-Allemagne et des sujets inclus dans l'agenda bilatéral le long de la période 2005-2018. La recherche identifie deux priorités communes: 1) la promotion des rapports économiques et commerciaux à l'aide du pragmatisme (même aux dépens du dialogue concernant les principes de la bonne gouvernance) et 2) l'institutionnalisation de l'agenda pour la coopération bilatérale, fondée sur une association stratégique intégrale des deux pays. L'essor des liens d'interdépendance économique est essentiel au renforcement de la coopération pour l'association stratégique. Celle-ci est un terrain institutionnel permettant d'accommoder des préférences et des intérêts divers (par exemple, l'engagement de l'Allemagne avec les relations transatlantiques, ou encore certains principes fondamentaux de la politique étrangère de la Chine, tels que la défense de sa souveraineté).

Mots clefs: Association stratégique intégrale Chine-Allemagne, interdépendance, Union Européenne, institutionnalisme libéral, asymétries.

\section{Traducción de Bernardo Mabire}

Fecha de recepción: diciembre de 2018

Fecha de aceptación: junio de 2019 


\section{INTRODUCCIÓN}

$\mathrm{E}$ n la investigación se elabora un análisis del desarrollo de la relación entre la República Popular China y la República Federal de Alemania (en adelante China y Alemania) durante el periodo 2005-2018, es decir, durante los cuatro periodos de gobierno encabezados por Angela Merkel en Alemania y los gobiernos presididos en China por Hu Jintao (2002-2012) y Xi Jinping (2012). La investigación utiliza un marco teórico liberal-institucionalista, en el que se pone énfasis en el análisis de una relación de interdependencia económica que ha contribuido a mantener la estabilidad en la relación bilateral entre dos potencias económicas con consecuencias significativas para el desarrollo de las relaciones China-Unión Europea (UE) y para el sistema de comercio global en general.

En la investigación se identifica que, con la finalidad de profundizar en sus relaciones de interdependencia, ambos países han realizado un esfuerzo diplomático para superar las asimetrías políticas y normativas que caracterizan la relación, producto de los diferentes procesos de desarrollo histórico que han experimentado: por un lado, China, una potencia exportadora con características internas que la aproximan, todavía, a los países en vías de desarrollo, con un sistema político autoritario dominado por el Partido Comunista Chino (PCCh) y que tiene la defensa de su soberanía, de su integridad territorial y la no intervención de extranjeros en sus asuntos internos como principios estructurales de su política exterior. Por otro lado, Alemania, una potencia exportadora, generadora de productos y servicios de alto valor, con un sistema político pluralista y cuyos principios de política exterior incluyen claros compromisos históricos con sus aliados en Occidente y los valores del liberalismo económico.

El análisis del desarrollo de la relación China-Alemania durante los años 2005-2018 tuvo como un resultado inicial la identificación de dos etapas diferenciadas: 1) durante una primera etapa (2005-2009), la relación sino-alemana se ca- 
racterizó por un distanciamiento debido a las asimetrías políticas y normativas que caracterizan a ambos países; b) posteriormente, en una segunda etapa (2009-2018), la relación sino-alemana se ha desarrollado a través de una diplomacia económica pragmática, producto del proceso de crisis económica mundial desencadenado a partir de los años 2007-2008: el rasgo político estructural más significativo, producido por la etapa del acercamiento pragmático (que continúa hasta la actualidad), ha sido el proceso de institucionalización de la relación bilateral mediante la elevación del nivel del vínculo a una asociación estratégica integral China-Alemania, redefinido entre 2010 y 2014.

A partir de la redefinición de la asociación estratégica, la relación bilateral China-Alemania ha incrementado sus relaciones de cooperación política y económica. Esta asociación ha funcionado como un espacio para la generación de un discurso diplomático compartido sobre los objetivos, intereses y las prioridades que tienen en común ambos actores y también funciona como una plataforma institucional en la que se coordina su agenda de cooperación: las relaciones de interdependencia económica han funcionado como el principal incentivo para continuar con el proceso de institucionalización. ${ }^{2}$ En la investigación se tratará de definir el carácter estructural o coyuntural de las relaciones de cooperación China-Alemania: en caso de ser un proceso estructural, la redefinición de esta relación bilate-

${ }^{2}$ De acuerdo con Robert O. Keohane y Joseph S. Nye, "en política mundial, interdependencia se refiere a situaciones caracterizadas por efectos recíprocos entre países o entre actores en diferentes países [...]; las relaciones interdependientes siempre implicarán costos, dado que la interdependencia reduce la autonomía, pero es imposible determinar $a$ priori si los beneficios de una relación serán mayores que los costos. Esto dependerá tanto de los valores que animen a los actores como de la naturaleza de la relación”. R.O. Keohane y J.S. Nye, "La interdependencia en la política mundial" en A. Borja (comp.) Interdependencia, Cooperación y Globalismo. Ensayos escogidos de Robert O. Keohane, CIDE, México, 2005, pp. 101-103. 
ral puede representar una evidencia de un proceso más amplio de redistribución del poder mundial. En el trabajo se analiza la capacidad de los actores para coordinar y ampliar sus políticas de cooperación de acuerdo con las coyunturas de crisis que se han presentado en el sistema internacional durante el periodo de tiempo estudiado.

Con una finalidad metodológica, la investigación se divide en cuatro partes que coinciden con los periodos de gobierno de Angela Merkel: 1) En la primera parte del trabajo se analizan los antecedentes generales de la relación sinoalemana desarrollada desde los primeros años de la década de 2000 y, especialmente, durante el periodo 2005-2009. Durante estos últimos años, la relación sino-alemana se caracterizó por un proceso de distanciamiento producido por la introducción, por parte del gobierno de Merkel, de una agenda orientada hacia la promoción de los valores normativos de la buena gobernanza en China, como la democratización de su sistema político, las críticas constantes del gobierno alemán a la situación de los derechos humanos en aquel país (ejemplificadas por los señalamientos en contra de la represión de los movimientos de protesta en Tibet, en 2008, y en Xinjiang, en 2009) y por los acercamientos al líder tibetano Dalái Lama. 2) En la segunda parte del trabajo se analiza el periodo 2009-2013; esta fase se inició con el agravamiento de los efectos de la crisis económica mundial en la eurozona y la redefinición efectuada por el gobierno alemán de los términos de su relación con China: desde 2009, el gobierno de Merkel se orientó a tener como prioridad una diplomacia económica pragmática con Beijing, en la que se privilegió la continuidad de las relaciones económicas y comerciales sino-alemanas, evitando la generación de tensiones políticas, como las del periodo anterior. Este nuevo contexto fue aceptado plenamente por el gobierno de $\mathrm{Hu}$ Jintao. De acuerdo con las nuevas prioridades del gobierno alemán, la promoción de los valores normativos quedó subordinada a los intereses de la diplomacia económica. Asimismo, a partir de 2010, la relación sino-alemana instrumentó una mayor canti- 
dad de mecanismos de diálogo y cooperación mediante la redefinición de su acuerdo de asociación estratégica. 3) En la tercera parte se analiza el periodo 2013-2017, fase caracterizada por la consolidación de la agenda pragmática y un nuevo proceso de institucionalización de la relación bilateral, acordado por ambos actores, con la instauración de nuevos mecanismos de diálogo y cooperación como parte de su asociación estratégica integral (finalmente oficializada en 2014); en la investigación se analiza el contenido de la agenda de la asociación estratégica. 4) El cuarto periodo empieza en 2017 y se ha caracterizado por una coyuntura de crisis en la relación transatlántica debido al discurso de crítica enarbolado por el presidente de Estados Unidos (EE.UU.), Donald Trump, hacia Alemania en temas que abarcan desde lo comercial (el superávit alemán sobre la economía estadounidense o las críticas a las relaciones comerciales ruso-alemanas para la transportación y compra de gas natural) hasta lo político (la posición crítica de Trump a las políticas de inmigración y refugio del gobierno de Merkel o su apoyo al Brexit) y la seguridad (las presiones para que el gobierno alemán incremente sus aportaciones al presupuesto de la Organización del Tratado del Atlántico Norte, OTAN). ${ }^{3}$

Sin embargo, las tensiones generadas por el comportamiento del presidente Trump no han sido motivo suficiente para que el gobierno alemán modifique de manera significativa sus prioridades de respeto a los compromisos tradicionales existentes en las relaciones transatlánticas que Alemania ha sostenido desde el periodo de Guerra Fría (y que continuaron tras la reunificación en 1990). ${ }^{4}$ En el trabajo se argumenta que, no obstante las críticas de Trump, en ocasiones

${ }^{3}$ E. Langenbacher y R. Wittlinger, "The End of Memory? GermanAmerican Relations under Donald Trump”, German Politics, vol. 27, núm. 2 (2018), pp. 181-183.

${ }^{4}$ A. Bendiek, "The '2014 Review': Understanding the Pillars of German Foreign Policy and the Expectations of the rest of the World", Working Paper RD EU/Europe, núm. 2015/05, Berlín, Stiftung Wissenschaft und Politik, 2015, p. 6. 
dirigidas de manera personal en contra de la canciller Merkel, especialmente durante 2017, el gobierno alemán ha mantenido los principios estructurales de sus vínculos transatlánticos (por ej., la pertenencia a la OTAN, el respeto al derecho internacional y el apoyo al sistema económico de libre comercio) como sus principales preferencias con el objetivo de preservar la estabilidad del sistema internacional. Sin embargo, estos compromisos no resultan contradictorios para continuar con la profundización de sus relaciones de interdependencia económica con China. De la misma manera, la relación sino-alemana se presenta como un componente estructural fundamental para las relaciones China-uE en lo general y, por consiguiente, es de una relevancia notoria para la preservación de la estabilidad del sistema internacional.

Debe considerarse que, en la actualidad, Alemania es el principal socio comercial de China en Europa y que, para Alemania, China es su primer socio comercial. Durante 2018 el total de las transacciones comerciales sino-alemanas alcanzó 199300 millones de euros(aproximadamente un tercio de la totalidad de las relaciones comerciales China-ue, las cuales ascendieron a 604604 millones de euros en 2018), aunque con un déficit contrario a Alemania de 13000 millones de euros (en contraste, el superávit comercial alemán con EE.UU. ascendió a 49000 millones de euros en 2018)..$^{5}$ En el contexto de la profundización de la interdependencia económica en la relación sino-alemana (aunque no exenta de

${ }^{5}$ Statistisches Bundesamt, "China is Germany's main Trading Partner once again" (comunicado de prensa), Destatis, Wiesbaden, 18 de febrero de 2019, https://www.destatis.de/EN/Press/2019/02/PE19_057_51.html; Dirección General de Comercio de la Comisión Europea, European Union, Trade in goods with China, Bruselas, 6 de marzo de 2019, https://webgate. ec.europa.eu/isdb_results/factsheets / country/details_china_en.pdf; Rene Wagner, "Exclusive: German Trade Surplus with U.S. declined in 2018 but was still almost 49 Billion Euros", Reuters, 11 de febrero de 2019, https:/ / www.reuters.com/article/us-germany-economy-exclusive/exclu sive-german-trade-surplus-with-u-s-declined-in-2018-but-was-still-almost49-billion-euros-idUSKCN1Q01EY 
sus propias tensiones, como el incremento del déficit alemán frente a China, la negativa de la UE a otorgar el estatus de economía de mercado [EME] a China y las acusaciones europeas de dumping, así como de violaciones a los derechos de autor en contra de empresas chinas), la aparición de un discurso proteccionista y antieuropeísta, como el de Trump, inoportuno, por lo menos, para los intereses de EE.UU. y la UE: hasta el momento, la diplomacia alemana ha hecho un esfuerzo para mantener la estabilidad en la relación transatlántica, en forma coherente con las características de los cuatro periodos de gobierno de Angela Merkel, si bien en un contexto en que la interdependencia sino-alemana se profundiza.

EL PRIMER PERIODO (2005-2009): ASIMETRÍAS NORMATIVAS Y DIFERENDOS COMERCIALES

En esta primera parte de la investigación, se elabora una descripción de los antecedentes generales de la relación sinoalemana previos a 2005 y se analizan las características de la relación durante el primer gobierno de coalición encabezado por Angela Merkel (2005-2009). Es necesario mencionar que una particularidad del sistema político alemán, con un régimen de gobierno parlamentario, reside en que sus gobiernos ejecutivos suelen estar integrados por coaliciones debido a la imposibilidad del partido mayoritario de ganar las elecciones con la cantidad suficiente de representantes para establecer una mayoría absoluta en Parlamento Federal Alemán (Bundestag). ${ }^{6}$

Los cuatro periodos de gobierno de Merkel (2005-2018) se han caracterizado por la formación de coaliciones entre la Unión Demócrata Cristiana-Unión Social Cristiana de Baviera (UDC/USC, unión de partidos conservadores a la que pertenece la canciller) con otros partidos de oposición que tienen

${ }^{6}$ B. Miller y W. Müller, "Managing Grand Coalitions: Germany 200509”, German Politics, vol. 19, núm. 3-4, 2010, pp. 334-337. 
presencia mayoritaria en el parlamento federal: es decir, en ninguno de sus cuatro periodos la canciller Merkel ha gobernado con una mayoría parlamentaria absoluta. ${ }^{7}$ Es importante tomar en cuenta estas características debido a que los ministerios del gobierno alemán pueden estar ocupados por una personalidad de diferente orientación política a la UDC/USC: algo relevante para la diplomacia alemana en términos políticos, económicos y de cooperación para el desarrollo. Las cuatro coaliciones formadas durante los periodos de gobierno de Merkel han sido las siguientes, en orden sucesivo: 1) 2005-2009: UdC/USC-Partido Socialdemócrata de Alemania (PSD); 2) 2009-2013: UDC/UsC-Partido Democrático Libre (FDP, por sus siglas en alemán); 3) 2013-2017: UDC/ USC-PSD; 4) desde 2017: UDC/USC-PSD. ${ }^{8}$

Algunos antecedentes de la relación sino-alemana: el pragmatismo económico del gobierno de Gerhard Schroeder (1998-2005)

El desarrollo de la relación sino-alemana durante los años previos al inicio del primer gobierno de la canciller Merkel se caracterizó por un acercamiento constante en las posiciones entre los gobiernos alemán y chino sobre diversas cuestiones de la actualidad internacional de aquel momento. El gobierno de Gerhard Schroeder (1998-2005, perteneciente al PSD) había instrumentado, desde su primer año, una política de aproximación pragmática hacia China en la que privilegiaba la cooperación económica sino-alemana, de esta forma, en 1999 realizó dos visitas oficiales a China, en mayo y noviem-

${ }^{7}$ Cuando la coalición está formada por los dos partidos políticos mayoritarios del sistema político alemán (UDC/USC y PSD) recibe el nombre de Gran Coalición.

${ }^{8}$ Los siguientes funcionarios han ocupado el cargo de ministro federal de Asuntos Exteriores durante los cuatro periodos de gobierno de Merkel: Frank-Walter Steinmeier, del PSD (2005-2009); Guido Westerwelle, del FDP (2009-2013); Frank-Walter Steinmeier, del PSD (2013-2017); Sigmar Gabriel del PSD (2017-2018) y Heiko Maas del PSD (2018). 
bre. ${ }^{9}$ La justificación de la agenda del gobierno alemán sobre China residió en su creciente importancia económica (como resultado del proyecto de modernización chino iniciado en 1978): el país asiático fue considerado un mercado clave para la economía alemana y sus empresas (desde ese entonces muy dependientes del sector exportador). ${ }^{10}$ Además, dos aspectos contribuyeron a facilitar la cordialidad en la relación bilateral: 1) por un lado, la ausencia de una rivalidad geopolítica entre ambas potencias, ubicadas en espacios geográficos distantes; 2) por otro lado, la ausencia de una memoria histórica en la República Popular que considerara a Alemania como un país imperialista y agresor en contra del territorio chino (al contrario de lo que ocurre con EE.uU., Francia y el Reino Unido). Los últimos acontecimientos significativos de un conflicto sino-alemán ocurrieron cuando las fuerzas alemanas participaron en la represión de la rebelión bóxer entre 1900-1901, y el último Puerto de Tratado ${ }^{11}$ perteneciente al Imperio alemán, en Shandong, fue desocupado en $1918 .{ }^{12}$

${ }^{9}$ El gobierno de Gerhard Schroeder estuvo formado por la Coalición PSD-Alianza 90/Los Verdes (Bündnis 90/Die Grünen). El ministro federal de Asuntos Exteriores durante el gobierno de Schroeder fue Joschka Fischer (1998-2005).

${ }^{10}$ E. Sandschneider, "China's Diplomatic Relations with the States of Europe”, China Quarterly, núm. 169 (2002), p. 38.

11 "Se denomina como Puertos de Tratado a las zonas portuarias abiertas al comercio con el exterior por las potencias extranjeras en China (principalmente europeas además de Estados Unidos y Japón) mediante los llamados "tratados desiguales" durante el siglo xIx y la primera mitad del xx. Los primeros Puertos de Tratado en China fueron abiertos con la conclusión de la Primera Guerra del Opio, mediante la firma del Tratado de Nanjing en 1842, entre el Imperio británico y la Dinastía Qing. Como consecuencia de su derrota, el gobierno Qing se comprometió a pagar indemnizaciones por la guerra, a la cesión de Hong Kong a perpetuidad a los ingleses y abrir al comercio con el exterior a los puertos de Cantón, Shanghái, Amoy, Ningbo y Fuzhou". Véase Marisela Connelly, "Comercio y consumo de opio en China”, Estudios de Asia y África, vol. 25, núm. 3 (83), 1990, p. 399.

${ }^{12}$ H. Kundnani y J. Parello-Plesner, "China and Germany: Why the Emerging Special Relationship matters for Europe”, Policy Brief núm. 55, Londres, ECFR (2012), p. 4. El primer acuerdo comercial entre la Repúbli- 
Igualmente, el gobierno de Schroeder se había atribuido prerrogativas mediante las cuales la cancillería alemana desarrollaba una agenda de diálogo directa con el gobierno chino (encabezado entonces por el presidente Jiang Zemin y el primer ministro Zhu Rongji) de manera suplementaria a la agenda del Ministerio Federal de Asuntos Exteriores alemán. ${ }^{13}$ Como señala Joern-Carsten Gottwald:

Desde hace muchos años, la política alemana hacia China ha sido tarea del canciller alemán. La reducción de la influencia de Ministerio Federal de Asuntos Exteriores, con la finalidad de priorizar la cooperación económica, continúa con un rumbo tradicional trazado por otros gobiernos de Alemania. De esta manera, las relaciones bilaterales entre un Berlín democrático y un Beijing autoritario alcanzan un nivel asombroso de cercanía política y personal. El gobierno de Schroeder estableció una cierta división del trabajo entre el canciller, como promotor del comercio, y los otros miembros del Ejecutivo, como críticos morales ocasionales, señalando la pobreza del historial

ca Popular China y Alemania Occidental entró en vigor en 1957, aunque ambos países establecieron relaciones diplomáticas oficiales hasta 1972. Durante la Guerra Fría, las relaciones económicas bilaterales tendieron a aumentar de manera constante, así, durante la década de 1980, Alemania se convirtió en el principal socio comercial de China de entre los miembros de la Comunidad Económica Europea (CEE). Las relaciones económicas se interrumpieron temporalmente entre 1989-1992 como consecuencia de las sanciones europeas derivadas por la represión en la Plaza Tiananmen en 1989. Sin embargo, al finalizar la década de 1990, el volumen del comercio bilateral sino-alemán era más alto que "el comercio combinado de China con Francia, Reino Unido e Italia”. K. Rupprecht, "German's Policy toward China and the SARs of Hong Kong and Macau”, en Miguel Santos-Neves y Brian Bridges (ed.), Europe, China and the two Sars: towards a New Era, Londres, Macmillan Press, 2000, p. 65.

13 F. Heiduk, "Conflicting Images? Germany and the Rise of China", German Politics, vol. 23, núm. 1-2 (2014), p. 118; P.K. Wu y M. D. Jensen, "Examining the EU-China Relationship in the Aftermath of the Economic Crisis", International Journal of Public Administration, vol. 40, núm. 14 (2017), p. 1231. 
de derechos humanos de China y su renuencia a introducir reformas políticas esenciales. ${ }^{14}$

Como un ejemplo de esta relación cercana, en 1999, durante la intervención militar a Serbia, la OTAN bombardeó las instalaciones de la embajada de China en Belgrado (a decir del gobierno de Bill Clinton, de manera "accidental"): el representante de la UE encargado de presentar una disculpa personal al gobierno chino en Beijing fue el canciller Schroeder. ${ }^{15}$ En 1999, el gobierno alemán también estableció dos diálogos de índole normativa con el gobierno chino: el Diálogo sobre derechos humanos y un Diálogo sobre el Estado de derecho (que continúan efectuándose hasta la actualidad); sin embargo, la prioridad en la agenda bilateral fue otorgada al Diálogo jurídico: diseñado entonces como un proceso de cooperación de larga duración "con el objetivo de mejorar las prácticas legales y judiciales en China”. Este Diálogo combina elementos tales como una crítica pública cuidadosa, la colaboración en proyectos específicos, mantiene conversaciones a puertas cerradas y una cooperación pragmática. ${ }^{16}$

Por su parte, desde la década de 1990, el gobierno chino había puesto en valor a Alemania por su capacidad de liderazgo en la orientación de algunas políticas de la UE (especialmente en el aspecto económico y comercial), así como por la condición alemana de primera potencia económica europea.

${ }^{14}$ J.C. Gottwald, "Germany's China-Policy: Trade Promotion, Human Rights and European Disunity", en M. Overhaus et. al. (eds.), GermanChinese Relations: Trade Promotion Plus Something Else?, German Foreign Policy in Dialogue vol. 6, núm. 16 (2005), p. 7.

${ }^{15}$ C. Schnellbach y J. Man, "Germany and China: Embracing a Different Kind of Partnership?", CAP Working Paper, Múnich, CAP, University of Munich, 2015, p. 2; J.-C. Gottwald, art. cit. p. 8.

${ }^{16}$ Ibid., p. 9. En septiembre de 2000 ambos gobiernos firmaron un Acuerdo de Intercambio y Cooperación Jurídica con la finalidad de institucionalizar el Diálogo sobre Estado de Derecho. Véase Ministerio Federal de Justicia y Protección al Consumidor, The German-Chinese Rule of Law Dialogue, Berlín, https://www.bmjv.de/EN/EU/Topics/GermanChineseLawDialogue/GermanChineseLawDialogue_node.html 
Desde esta perspectiva, Alemania se presentó a los intereses de China como un "puente" de acceso ineludible para tener presencia en el espacio europeo. El comportamiento pragmático de la diplomacia alemana también fue valorada de forma positiva por el gobierno chino: 1) por un lado, Alemania ha sido considerado un país realista, con posiciones frecuentemente amistosas hacia China, y con una clara capacidad para influir en el entorno europeo e internacional; 2) algunas políticas de desarrollo económico adoptadas por el gobierno alemán se consideran ejemplares por el gobierno chino, específicamente en temas como la organización industrial, programas de innovación tecnológica, seguridad social, mercados financieros, entrenamiento vocacional, etcétera. ${ }^{17}$

La relación de cercanía entre los poderes ejecutivos chino y alemán en términos económicos, durante el gobierno de Schroeder, se profundizó mediante el incremento de las relaciones comerciales sino-alemanas, el apoyo de Alemania (y de la Ue) a la incorporación de China a la Organización Mundial del Comercio (omc) en 2001 y las diversas actividades de cooperación científica y tecnológica instrumentadas en la relación bilateral. La justificación del gobierno alemán para subordinar la agenda de los valores normativos a los intereses económicos residió en que mediante el incremento de las relaciones comerciales, China podía ser integrada a las normas del sistema internacional y, por lo tanto, podría contribuir con su transformación interna: esta política recibió la denominación de "Wandel durch Handel", es decir, "cambio a través del comercio". Así lo afirmaba Schroeder: "El 'intercambio económico' daría lugar al 'cambio social' ". De esta forma, el gobierno socialdemócrata tendió a enfatizar la cooperación en lugar de la confrontación con China. ${ }^{18}$

17 M. Huotari, "Germany's China Policy: No Honeymoon Forever", en M. Huotari, et. al. (eds.), Mapping Europe-China Relations. A Bottom-Up Approach, Berlín, MERICS, 2015, p 31, https://www.ifri.org/sites/default/ files/atoms/files/etnc_web_final_1-1.pdf

18 G. Schroeder, citado en H. Kundnani y J. Parello-Plesner, op. cit. p. 3 . 
A las relaciones pragmáticas en el ámbito de la economía se sumaron algunas coincidencias políticas, especialmente a partir de 2003: en ese momento, los gobiernos de Schroeder y de Jacques Chirac, en Francia, se opusieron en la Organización de Naciones Unidas (ONU) a los planes del gobierno de George W. Bush para invadir Irak (provocando un acercamiento con la posiciones de China y Rusia). ${ }^{19}$ En un contexto contrario al unilateralismo del gobierno de Bush (y de distanciamiento en las relaciones transatlánticas del eje francoalemán), en octubre de 2003, la uE y China formalizaron un acuerdo de asociación estratégica y, posteriormente, en 2004, la relación sino-alemana anunció el establecimiento de una "asociación estratégica con responsabilidades globales". ${ }^{20} \mathrm{De}$ acuerdo con Ieva Gajauskaité:

Las asociaciones estratégicas se caracterizan por ser una cooperación voluntaria y flexible basada en un compromiso mutuo formal. Por lo tanto, esta forma de cooperación tiene una amplia gama de aplicaciones y puede ser definida como el proceso de una cooperación internacional formalizada, estructurada e institucionalizada a fin de lograr los objetivos estratégicos comunes que surgen de los intereses nacionales y el deseo de incrementar la seguridad nacional. ${ }^{21}$

De la misma manera, en diciembre de 2003, durante una visita oficial a Beijing, el canciller Schroeder hizo un llamamiento al levantamiento del embargo de armas de la uE en

${ }^{19}$ J.-P. Cabestan, "La politique étrangère chinoise : une Chine sans ennemis n'est pas forcément une Chine rassurante", Hérodote, núm. 125 (2007), p. 16; H. Védrine, "L'Émergence de la Chine vue de France", Politique Étrangère, núm. 3 (2006), p. 485.

${ }^{20}$ C. Schnellbach y J. Man, op. cit. p. 5; H. Kundnani y J. Parello-Plesner, op. cit. p. 6.

${ }^{21}$ I. Gajauskaitè, "Strategic Partnerships in Foreign Policy: Comparative Analysis of Polish -Ukrainian and Lithuanian - Ukrainian Strategic Partnerships", Lithuanian Annual Strategic Review, vol. 11, núm. 1 (2013), p. 194. 
contra de China y, durante los siguientes dos años, Alemania impulsó en el Consejo Europeo un debate para el levantamiento del embargo (vigente desde 1989, como parte de las sanciones económicas derivadas de la represión en la Plaza Tiananmen). ${ }^{22}$ Con estas acciones, el gobierno de Schroeder mostraba también un claro cálculo económico sobre los beneficios que podrían obtener las economías de la UE, especialmente la alemana, por el levantamiento de las restricciones en contra de la comercialización de los productos tecnológicos relacionados con el mercado de armas hacia China. ${ }^{23}$

La relación sino-alemana durante el primer periodo de gobierno de Angela Merkel (2005-2009)

El inicio del primer periodo de gobierno de Merkel, en 2005, cambió el contexto de la relación sino-alemana desarrollado durante el gobierno de Schroeder (en términos generales, algo extensivo a la relación China-UE). El primer cambio del gobierno de Merkel fue una reaproximación con el gobierno de Bush para finalizar el periodo de distanciamiento transatlántico (el presidente Bush había ganado su reelección en 2004, condición que lo dotó de legitimidad para reiniciar un diálogo con los países de la ue que se habían opuesto a la invasión de Irak), con la consecuencia de la suspensión del debate sobre el levantamiento al embargo de armas en contra de China (al cual se oponían Ee.uu., el Parlamento Europeo y algunos miembros de la Ue, como Reino Unido y Polonia, que presionaban así al gobierno de Schroeder en sus últimos

22 "Schröder calls for end to Arms Embargo against China", Deutsche Welle, Beijing, 3 de diciembre de 2003, https://www.dw.com/en/schr\%C 3\%B6der-calls-for-end-to-arms-embargo-against-china/a-1047143

23 T. Grosse, "Geoeconomic Relations between the EU and China: The Lessons from the EU Weapon Embargo and from Galileo”, Geopolitics, vol. 19, núm.1 (2014), pp. 53-54. 
meses).$^{24}$ Aunque el mayor cambio fue la renovada importancia que se confirió a la cuestión de la situación de los derechos humanos en China y a la democratización del sistema político chino en la agenda de la relación bilateral (temas que el gobierno del Pcch puede considerar violatorios de la soberanía). ${ }^{25}$

A las asimetrías normativas derivadas de los términos del nuevo diálogo político sino-alemán, impulsado por Merkel, se sumó un periodo de tensiones en la relación económica China-ue, producto del creciente déficit comercial que comenzaba a experimentar la relación sino-europea (en 2006, el déficit comercial en la relación China-uE ascendió a 138571 millones de euros y en 2007 a 165974 millones). ${ }^{26}$ El conjunto de las economías europeas, en general, tenían una relación comercial deficitaria con China, especialmente en el caso de las economías con sectores exportadores cuya dependencia de industrias manufactureras como los textiles y el calzado era mayor (como España, Francia e Italia): en 2006 se gestó un importante debate crítico en las instituciones de la UE en contra de China debido a la introducción de manufacturas baratas chinas que ponían en peligro a las industrias europeas menos competitivas en cuanto a los costos. ${ }^{27}$ Las asimetrías normativas y las críticas a un co-

${ }^{24}$ O. Bräuner, "Beyond the Arms Embargo: EU Transfers of Defense and Dual-Use Technologies to China", Journal of East Asian Studies, vol. 13, núm. 1 (2013), pp. 462-463; J.-C. Gottwald, art. cit., p. 7; W. Crotty, "Armageddon, just another Campaign or something in between. The Meaning and Consequences of the 2004 Presidential Election" en W. Crotty (ed.) A Defining Moment: The Presidential Election of 2004, Routledge, Nueva York, 2015, pp. 237-238.

${ }^{25}$ C. Schnellbach y J. Man, op. cit., pp. 8-9.

${ }^{26}$ Dirección General de Comercio de la Comisión Europea, European Union, Trade in Goods with China, Bruselas, 16 de noviembre de 2017, p. 3, http://trade.ec.europa.eu/doclib/docs/2011/january/tradoc_147241. pdf

${ }^{27}$ N. Casarini, "The Evolution of the EU-China Relationship: from Constructive Engagement to Strategic Partnership", Ocassional Paper, núm. 64, París, Euiss, 2006, pp. 16-18; X. Zhang, EU-China Economic Diplomacy: 
mercio percibido como injusto caracterizaron las relaciones China-uE durante el periodo 2006-2008, y este proceso se extendió a la relación sino-alemana.

En septiembre de 2007, el gobierno de Merkel recibió al Dalái Lama en Berlín, lo que agravó las tensiones, y el gobierno alemán protestó de manera enérgica en contra de la represión del gobierno chino hacia las protestas en Tibet en 2008 y (de forma más moderada) en Xinjiang en 2009 (como parte de sanciones simbólicas, Merkel decidió no asistir a la inauguración de los Juegos Olímpicos de Beijing de 2008). ${ }^{28}$ Sin embargo, también como expresión de la importancia de las relaciones económicas bilaterales, el gobierno chino no se mostró tan molesto con Alemania como en el caso del anuncio de un encuentro del presidente francés Nicolas Sarkozy con el Dalái Lama en Gdansk, en diciembre de 2008 (ese año, el gobierno chino anunció la cancelación de la $11^{\circ}$ Cumbre China-ue que debía celebrarse en noviembre en Lyon debido a que Francia ejercía la presidencia semestral del Consejo Europeo); empero, en 2007 fue suspendido el Diálogo sino-alemán sobre derechos humanos. ${ }^{29}$ Hacia finales de 2008 parecía claro un empeoramiento en las tensiones de la relación sino-alemana (extensivas a la relación China-uE) hasta que en septiembre de ese año fue evidente la gravedad de la crisis económica mundial a partir de la quiebra de Lehman Brothers y el hundimiento de los mercados financieros internacionales. El nuevo contexto de la crisis

When Economics meets Politics, Wuhan, University Research Centre for Economic Diplomacy, Wuhan University, 2014, http:/ / ced.whu.edu.cn/Show / $\mathrm{id}=103 \&$ siteid=3

${ }^{28}$ C. Schnellbach y J. Man, op. cit. pp. 10-11; F. Heiduk, 2014, art. cit. pp. 118-119.

${ }^{29}$ A. Cunha, "Paving the New Silk Road: The Evolution of the SinoGerman Strategic Partnership”, Working Paper núm. 67, Observatorio Político, Lisboa, 2017, p. 10, http:/ /www.observatoriopolitico.pt/wp-content /uploads/2017/01/WP_67_AC.pdf; "China Cancels Human Rights Meeting After Dalai Lama Row”, Deutsche Welle, Berlín, 14 de octubre de 2007, https:/ / www.dw.com/en/china-cancels-human-rights-meeting-after-da lai-lama-row/a-2824545 
contribuyó a que el gobierno alemán redefiniera sus prioridades en la relación con China. ${ }^{30}$

\author{
EL SEGUNDO PERIODO (2009-2013): LA ESTRUCTURACIÓN \\ DE UNA RELACIÓN PRAGMÁTICA Y LA SUBORDINACIÓN DE \\ LOS VALORES NORMATIVOS
}

Como menciona Hans Kundnani, la creciente importancia del sector exportador para la economía de Alemania se vio impulsada por las reformas económicas instrumentadas especialmente en 2003 por el gobierno de Schroeder (con la finalidad de incrementar la competitividad de las empresas alemanas en el contexto de la introducción del euro en 2002). ${ }^{31}$ El proceso de dependencia de las exportaciones se habría acelerado durante la década del 2000 (durante los años previos al inicio de la crisis) y, de manera significativa, también incluyó algunos mercados no occidentales importadores de productos alemanes (como China e India). De acuerdo con datos del Banco Mundial, la contribución de las exportaciones alemanas a su producto interno bruto (PIB) aumentó de 33\% en 2000 a 48\% en 2010: "Alemania comenzó a enfocar su política exterior, de forma amplia, en el beneficio de sus intereses económicos y, en particular, en las necesidades de sus exportadores". ${ }^{32}$ Durante la segunda década del 2000, China se convirtió en el segundo mercado para las exportaciones alemanas entre los terceros países externos a la UE (de los cuales, EE.UU. ocupaba el primer lugar): como ejemplo, en la actualidad China es el principal mercado para

${ }^{30}$ C. Schnellbach y J. Man, op. cit. pp. 11-12; A. Cunha, op. cit. p. 11.

${ }^{31}$ Las reformas económicas de 2003 en Alemania se denominaron Agenda 2010 y consistieron en subvenciones al servicio del empleo con formación profesional, recortes a los subsidios del desempleo para reducir el de larga duración, el aumento de los contratos de trabajo temporales (los llamados minijobs), etcétera.

${ }^{32}$ H. Kundnani, "Leaving the West. Germany looks East", Foreign Affairs, enero-febrero, 2015, p. 110. 
marcas automotrices alemanas como Volkswagen y Mercedes Benz Clase S. ${ }^{33}$ Estas características estructurales de la economía alemana y la importancia de China como un mercado para sus productos determinaron la actual orientación pragmática de la relación. ${ }^{34}$ Desde una perspectiva crítica, la “excesiva preocupación” de la agenda económica por la diplomacia alemana ha tenido como consecuencia "un enfoque reducido sobre los intereses alemanes, poca capacidad para diseñar y dirigir proyectos [...] y una 'economización de la política exterior alemana". 35

Durante el segundo gobierno de Merkel (2009-2013) continuó la profundización de la interdependencia económica sino-alemana, ahora en el contexto de la crisis: la respuesta de ambos gobiernos fue desarrollar una tendencia hacia la institucionalización de los mecanismos de cooperación que habían establecido; desde la perspectiva alemana, la agenda bilateral se estructuró sobre tres prioridades básicas: 1) impulsar la liberalización del mercado interno de China de forma gradual; 2) incorporar a China en el sistema del derecho internacional; 3) en caso de conflictos, la preferencia del gobierno alemán sería la salvaguarda de los intereses económicos de las empresas alemanas (especialmente de su sector exportador a China). ${ }^{36}$ De la misma forma, como parte de la reaproximación en la relación China-ue, en mayo de 2009 se realizó la $11^{\circ}$ Cumbre China-ue en Praga (que se había suspendido en 2008) y en noviembre de 2009 se efectúo la $12^{\circ}$ Cumbre China-ue en Nanjing: en el contenido de los Comunicados conjuntos de ambas cumbres se reflejaron las nuevas prioridades de la agenda sino-europea, basadas ahora en el pragmatismo

33 Ibid. p. 114.

${ }^{34}$ P.-K. Wu y M. D. Jensen, art. cit., p. 1231; F. Godement, "Key issues of China's Leadership Succession and their Relevance to the EU", en N. Casarini, (ed.), Brussels-Beijing: changing the game? Report núm. 14, París, EUISs, 2013, p. 65, https://www.files.ethz.ch/isn/182364/Report_14.pdf

35 A. Bendiek, op. cit., p. 6.

${ }^{36}$ M. Huotari, op. cit., p. 31. 
económico, las cuales también fueron compartidas por el gobierno alemán (aunque en detrimento de la agenda de la buena gobernanza). En 2010 se oficializó al pragmatismo económico como la base estructural de la relación sino-europea (y sino-alemana). ${ }^{37}$

La redefinición de la relación sino-alemana, impulsada por el gobierno de Merkel, dio inicio formalmente durante su visita oficial a Beijing en julio de 2010: entonces, la canciller propuso una mayor institucionalización de las relaciones mediante la celebración, de manera formal, de una cumbre anual sino-alemana, al mismo tiempo que la asociación estratégica sería elevada al nivel de una "asociación estratégica integral”, agregando nuevas actividades bilaterales (la agenda completa de la asociación estratégica fue oficializada en 2014). ${ }^{38}$ La Cumbre China-Alemania podría celebrarse anualmente de forma paralela a otros eventos internacionales a los que pudieran asistir, de manera eventual, la canciller y el ejecutivo chino (como la Cumbre China-ue y las cumbres del G-20, adicionando así los temas multilaterales a la agenda bilateral en temas como la economía y la seguridad). Durante la Cumbre China-Alemania de 2010, Merkel declaró: "China, con un rápido crecimiento económico, es un socio importante de Alemania y Europa [...]. Alemania está lista para trabajar con China para ampliar y profundizar la cooperación bilateral

37 Consejo Europeo, 11th EU-China Summit Prague Joint Press Communiqué 10234/09 (Presse 147), Bruselas, 20 de mayo de 2009, https:/ / ec.europa.eu/clima/sites/clima/files/docs/0023/joint_statement_en.pdf; Ministerio de Asuntos Exteriores de China, Joint Statement of the 12th China-EU Summit, Nanjing, 30 de noviembre de 2009, http://www.chinamission.be/eng/zywj/zywd/t1228338.htm

38 "China, Germany to hold Annual Consultations to deepen 'Strategic Partnership', Deutsche Welle, 16 de julio de 2010, http://www.dw.de/ china-germany-to-hold-annual-consultations-to-deepen-strategicpartners hip/a-5809459; Ministerio de Asuntos Exteriores de China, Hu Jintao meets with German Chancellor Merkel, Beijing, 16 de julio de 2010, http://www. china-embassy.org/eng/zgyw/t717842.htm 
en política, economía, comercio, cultura, medio ambiente y asuntos multilaterales". 39

De igual manera, otros temas abordados por Merkel fueron la petición de una mayor apertura del mercado interno chino para la empresas alemanas (en reciprocidad a la apertura del mercado alemán a las inversiones chinas) y la invitación al gobierno y los capitales privados chinos a que invirtieran en el mercado financiero europeo (en el contexto de la grave crisis en la eurozona). ${ }^{40}$ Además de los intereses económicos, durante los primeros años de la crisis en la UE, los gobiernos chino y alemán encontraron que compartían algunas posturas similares en los debates multilaterales sobre la situación de la economía mundial (en foros como el G-20): ambos favorecieron la instrumentación de políticas deflacionarias (criticadas por EE.UU., pues éstas aumentaban el déficit con sus socios comerciales), además de que criticaron la expansión cuantitativa instrumentada por el gobierno de $\mathrm{Ba}-$ rack Obama. ${ }^{41}$

Durante la visita de Merkel de 2010, y como resultado de la redefinición de la asociación estratégica, China y Alemania incrementaron su agenda de cooperación política mediante la realización de diálogos consultivos de tipo intergubernamental en el nivel ministerial. La primera reunión del Diálogo Intergubernamental se llevó a cabo en junio de 2011 (con la conclusión de 22 acuerdos) y la segunda reunión se celebró en agosto de 2012. Estas reuniones tienen la forma de encuentros entre los gabinetes de ambos gobiernos y se han efectuado alternativamente en Alemania y China (se trata de cumbres de alto nivel, las primeras en su tipo en ser conduci-

${ }^{39}$ Loc. cit.

40 "Merkel pushes China to open its Markets", The Local, 16 de julio de 2010, https:/ /www.thelocal.de/20100716/28545

${ }^{41}$ H. Kundnani, 2015, art. cit., p. 115; I. Talley y J. Sparshott, "U.S. Blasts Germany's Economic Policies", The Wall Street Journal, 31 de octubre de 2013, https:/ / www.wsj.com/articles/us-treasury-targets-german-econo mic-policies-1383170134 
das por China con un miembro de la UE). ${ }^{42}$ Las áreas prioritarias acordadas en las consultas incluyen temas como la energía, el cambio climático, la protección del medio ambiente, la cooperación para el fortalecimiento del Estado de derecho en China y la cooperación para la innovación científica y tecnológica. En 2012, durante la Cumbre de negocios sinoalemana, el entonces primer ministro chino, Wen Jiabao, mencionó que en ese momento el comercio sino-alemán ascendía a 30\% del total de las relaciones comerciales China-uE. Además, la cooperación tecnológica bilateral ofrecía como resultados la instalación de un ecoparque sino-alemán en Qingdao y una plataforma estratégica para la movilidad en vehículos eléctricos, además de colaborar en la construcción de un portal de información para la estandarización industrial y uno para las ciencias de la vida. ${ }^{43}$

EL TERCER PERIODO (2013-2017): LA REDEFINICIÓN DE LA ASOCIACIÓN ESTRATÉGICA INTEGRAL SINO-ALEMANA

Durante el periodo 2013-2014 coincidieron dos procesos de transición política en China y Alemania, los cuales también incluyeron modificaciones en la instrumentación de sus respectivas políticas exteriores: 1) en el caso de China, el nuevo gobierno encabezado por Xi Jinping, desde la celebración del XviII Congreso del PCCh en noviembre de 2012, instrumentó un conjunto de reformas tendientes a fortalecer la centralización del poder político y económico en la figura

${ }^{42}$ H. Kundnani, 2015, art. cit., p. 115; Cancillería Federal de Alemania, Germany/China: stepping up close Cooperation, Berlín, 26 de mayo de 2013, https://www.bundeskanzlerin.de/ContentArchiv/EN/Archiv17/ Artikel/2013/05/2013-05-26-besuch-li-keqiang.html

${ }^{43}$ M. Huotari, op. cit., p. 30; Ministerio de Asuntos Exteriores de China, Address by H.E. Wen Jiabao Premier of the State Council of the People's Republic of China at the China-Germany Business Summit, Hannover, 23 de abril de 2012, http://www.fmprc.gov.cn/mfa_eng/topics_665678/wjbispg_66571 4/t930826.shtml 
del presidente chino (incluyendo la toma de decisiones en materia de política exterior). ${ }^{44}$ Desde un inicio, la política exterior de Xi se enfrentó a un aumento de tensiones por conflictos territoriales en Asia del Este (debido a las disputas por las islas Diaoyu-Senkaku con Japón y, en el Mar del Sur de China, con Filipinas y Vietnam). El gobierno chino atribuyó estas tensiones a políticas de contención generadas en su contra por la política de pivote hacia Asia del Este formulada por el gobierno de Obama en 2011: la respuesta de Beijing fue el incremento de sus relaciones de cooperación con sus socios ubicados en Eurasia, como en Asia central (por medio de la Organización de Cooperación de Shanghái ocs), con Rusia y la UE. Uno de los resultados de la política de "marchar hacia el oeste" del gobierno chino fue la presentación de la Iniciativa Cinturón y Ruta de la Seda en Astaná (ahora Nur-Sultán), Kazajistán, en septiembre de 2013. ${ }^{45}$ 2) En el caso de Alemania, en diciembre de 2013 comenzó el tercer periodo de gobierno de Angela Merkel: en esta ocasión estuvo integrado por una gran coalición y se designó a Frank-Walter Steinmeier (PSD) como el nuevo ministro federal de Asuntos Exteriores.

Como lo menciona Annegret Bendiek, hacia 2013-2014 el nuevo gobierno alemán se encontró con mayores responsabilidades en su posición de liderazgo en la UE y como potencia económica global (una de las pocas que se habían fortalecido durante el proceso de crisis económica) en temas como la continuación de la crisis en la eurozona, los conflictos en Medio Oriente (en 2011, Alemania había declinado su participación en la intervención de la OTAN en

${ }^{44}$ B. Naughton, "The Challenges of Economic Growth and Reform", en R. S. Ross y J. I. Bekkevold (eds.), China in the Era of Xi Jinping: Domestic and Foreign Policy Challenges, Washington, Georgetown University Press, 2016, pp. 78-82.

45 J. Wang, “ 'Marching Westwards': The Rebalancing of China's Geostrategy", en B. Shao (ed.), The World in 2020 According to China: Chinese Foreign Policy Elites discuss Emerging Trends in International Politics, Leiden, Brill, 2014, p. 131. 
Libia), la crisis diplomática con EE.UU. generada por el caso de espionaje de la Agencia de Seguridad Nacional (NSA, por sus siglas en inglés) o la crisis política que se comenzó a gestar en Ucrania a partir de noviembre de 2013 (al rechazar el gobierno ucraniano de Viktor Yanukóvich la firma de un acuerdo de asociación con la UE) ${ }^{46}$ Como respuesta al nuevo contexto internacional, en diciembre de 2013 el ministro Steinmeier dio inicio a un debate para elaborar una "revisión" de los "valores e intereses" de la política exterior alemana. ${ }^{47}$ De acuerdo con Bendiek, el debate sobre la política exterior durante el tercer gobierno de Merkel tuvo como resultado el proyecto denominado Revisión 2014: una mirada nueva a la política exterior alemana, articulado gracias la participación de expertos de think tanks como la German Marshall Fund y la Stiftung Wissenschaft und Politik. ${ }^{48}$

Como lo señala Zhao Ke, durante el periodo de crisis económica, la política exterior de Alemania se había mantenido en un "equilibrio precario", entre los beneficios que el proceso de globalización aportaba a su economía exportadora y el impulso que los gobiernos alemanes debían proporcionar al proceso de integración europeo (considerando las dificultades económicas y políticas que enfrentaba la integración). ${ }^{49}$ En primera instancia, había un contraste entre los beneficios

${ }^{46}$ A. Bendiek, op. cit. p. 4.

${ }^{47}$ F.-W. Steinmeier, Speech by Dr Frank-Walter Steinmeier, Federal Minister for Foreign Affairs, at the Handover Ceremony on 17 December 2013, Berlín, Ministerio Federal de Asuntos Exteriores de Alemania, 17 de diciembre de 2013, https:/ / www.auswaertiges-amt.de/en/newsroom/news/131217bm-antrittsrede/258766

48 A. Bendiek, op. cit. p. 4; Ministerio Federal de Asuntos Exteriores de Alemania, "Review 2014-A Fresh Look at German Foreign Policy" - Closing Remarks by Foreign Minister Frank-Walter Steinmeier, Berlín, 20 de mayo de 2014, https://www.auswaertiges-amt.de/en/newsroom/news/140520bm-review2014-abschlussrede/262346

${ }^{49}$ K. Zhao, "Germany's Diplomatic Rebalancing and Its Impact on China”, China International Studies, núm. 66, septiembre-octubre de 2017, https://www.pressreader.com/china/china-international-studies-engli $\mathrm{sh} / 20170920 / 281500751430764$ 
económicos para la economía alemana y sus empresas en comparación con las consecuencias de la crisis financiera en la eurozona a partir del periodo 2009-2010 y la intervención de la Troika (Banco Central Europeo, Fondo Monetario Internacional y Comisión Europea) a países como Grecia y Portugal. ${ }^{50}$ Para Zhao, a partir de 2014 estos procesos orientaron a la diplomacia alemana a efectuar un "reequilibrio" (como el autor denomina a uno de los objetivos del proyecto Revisión 2014), al mantener su compromiso con la continuidad de la integración europea, aunque sin comprometer los beneficios económicos para la economía alemana: "Un principio cardinal de la política exterior alemana es mantener el equilibrio" entre ambas dinámicas. Por el lado alemán, este contexto de equilibrio entre ambas prioridades es lo que ha determinado el escenario de "retos y oportunidades" para el desarrollo de las relaciones China-Alemania en los años posteriores. ${ }^{51}$

La condición de Alemania como beneficiaria de la globalización y garante de la UE orientó a Berlín a fortalecer sus preferencias por el respeto a las normas del derecho internacional y la continuidad del sistema económico de libre comercio: la política de reequilibrio buscaba "[...] profundizar la cooperación entre países desarrollados y construir un grupo institucionalizado político, económico y financiero de países desarrollados, promoviendo un 'Occidente más grande' ". ${ }^{52}$ Estas preferencias por la estabilidad del sistema internacional, de acuerdo con los principios de Alemania, se reafirmaron durante la 50 Conferencia de Seguridad de Múnich, realizada en enero de 2014; en ésta, las intervenciones de los entonces presidente federal alemán, Joachim Gauck, el ministro Steinmeier y la ministra federal de Defensa, Ursula von der Leyen, generaron un discurso en torno del así

50 Loc. cit.

51 Loc. cit.

${ }^{52}$ K. Zhao, "Does ascendant Germany pose a Challenge for China?", Chinese Social Sciences Today, Beijing, 3 de mayo de 2016, http:/ / english.cssn. cn/research/internationalstudies/201603/t20160308_2903111.shtml 
denominado “consenso de Múnich”, el cual subrayaba el equilibrio necesario entre los compromisos del gobierno alemán con la integración europea, la alianza transatlántica y la globalización económica. ${ }^{53}$ Durante su discurso en la Conferencia de Múnich, el presidente Gauck señaló:

Alemania ha demostrado desde hace mucho tiempo que actúa de forma internacionalmente responsable. Pero podría, tomando como base su experiencia en la protección de los derechos humanos y el Estado de derecho, adoptar medidas más resueltas para preservar y dar forma al orden basado en la Unión Europea, la OTAN y las Naciones Unidas. Al mismo tiempo, Alemania también debe estar preparada para hacer más para garantizar la seguridad que otros le han brindado durante décadas. ${ }^{54}$

Por su parte, el ministro Steinmeier enfatizó durante su intervención: "Durante las últimas décadas, Europa y EE.UU. se han mantenido muy cerca. Para nosotros, la alianza atlántica ha sido un ancla indispensable en un mundo con problemas. Tanto económica como políticamente, Europa y EE.UU. son los socios más cercanos". ${ }^{55}$ Como mencionan Terhalle y Giegerich, mediante la formulación de este "consenso de Múnich”, los funcionarios alemanes resaltaron un propósito estratégico: "Contribuir a reformar y defender activamente el orden internacional liberal que es la base de la prosperidad

${ }^{53}$ M. Terhalle y B. Giegerich, "The Purpose of the German Power", en J. Bindenagel, M. Herdegen y K. Kaiser (eds.), International Security in the $21^{\text {st }}$ Century. Germany's International Responsibilities, Bonn, University Press, 2017, pp. 243-244; K. Zhao, 2016, art. cit.

${ }^{54}$ J. Gauck, Speech to open 50 th Munich Security Conference "Germany's role in the world: Reflections on Responsibility, Norms and Alliances”, Múnich, 31 de enero de 2014, http://www.bundespraesident.de/SharedDocs/Reden/ EN/JoachimGauck/Reden/2014/140131-Munich-Security-Conference. html

${ }^{55}$ F.-W. Steinmeier, Speech by Foreign Minister Frank Walter Steinmeier at the 50th Munich Security Conference, Múnich, 31 de enero de 2014, https:// www.auswaertiges-amt.de/en/newsroom/news/140201-bm-muesiko/ 259556 
de Alemania”, al tiempo que acentuaban la continuación de los compromisos de la relación transatlántica como la base de este orden. ${ }^{56}$

Los principios contenidos en el "consenso de Múnich" fueron el contexto que determinó la profundización de las relaciones de interdependencia económica y cooperación en la relación sino-alemana, siempre con la condición de mantener el respeto al derecho internacional, el sistema de libre comercio y la salvaguardia de los intereses de la UE: para el gobierno alemán, éstas son las bases de la redefinición de la asociación estratégica sino-alemana de $2014 .{ }^{57}$ Las nuevas condiciones en Alemania se evidenciaron en la profundización de las relaciones económicas sino-alemanas en comparación con el contexto de ruptura y sanciones que han caracterizado las relaciones ruso-alemanas, a partir del mismo año (es decir, desde el conflicto político en Ucrania y la intervención rusa en Crimea y en las regiones donde se habla ruso de Ucrania oriental). ${ }^{58}$

Desde los años previos a 2014, la relación China-Alemania comenzó a ser denominada por algunos expertos y en medios de comunicación como una "relación especial": en 2013, las relaciones económicas bilaterales alcanzaron un récord de 141456 millones de euros y, como parte del proceso de internacionalización de la moneda china, el centro financiero de Frankfurt fue designado como el primer punto de cambio del yuan renminbi en la eurozona. ${ }^{59}$ Sin embargo, para el académi-

${ }^{56}$ M. Terhalle y B. Giegerich, op. cit. p. 244.

${ }^{57}$ Gobierno Federal de Alemania, Joint Declaration between Germany and China: Comprehensive Strategic Partnership, The Press and Information Office of the Federal Government, Berlín, 28 de marzo de 2014, https://www. bundesregierung.de/Content/EN/Pressemitteilungen/BPA/2014/201403-28-china-declaration.html

${ }^{58}$ M. Terhalle y B. Giegerich, op. cit. pp. 247-248; T. Forsberg, "From Ostpolitik to "Frostpolitik"? Merkel, Putin and German Foreign Policy towards Russia”, International Affairs, vol. 92, núm. 1 (2016), p. 24.

${ }^{59} \mathrm{Li}$ Wei, The Limits of the China-Germany Special Relationship, Beijing, Institute of European Studies, Chinese Academy of Social Sciences, 2014, http://ies.cass.cn/wz/mtcf/201404/t20140409_2463171.shtml; Statistis- 
co Yang Xiepu resultaba claro que esta "relación especial" sólo era otra manera de enunciar el pragmatismo económico: "Basado más en las relaciones económicas y comerciales que en los valores y sistemas". ${ }^{60}$ En marzo de 2014, el presidente Xi realizó visitas de Estado a Alemania y Bélgica con la finalidad establecer los nuevos términos de la asociación estratégica sino-alemana, así como para presentar los proyectos de inversión de la Iniciativa Cinturón y Ruta de la Seda en el puerto de Duisburgo (terminal del ferrocarril Yuxinou que recorre la ruta Chongqing-Duisburgo) y los principios básicos de la Iniciativa en la sede de la UE en Bruselas. ${ }^{61}$

De acuerdo con Zhao Ke, el establecimiento de una "relación especial" sino-alemana ha tenido como objetivo, para el gobierno chino, el de una "reducción de los costos de coordinación" en la política de Beijing hacia la uE, debido al papel de liderazgo que Alemania tiene en el espacio europeo $y$, desde la percepción china, la influencia positiva que puede tener el gobierno alemán en la coordinación de la política comunitaria hacia China. ${ }^{62}$ Sin embargo, el abuso en esta percepción puede generar una disonancia cognitiva sobre el alcance real de la influencia de Alemania en las políticas comerciales de la UE; como advierte Li Lezeng: "Es imposible para Alemania (cuya influencia es limitada) gestionar todas las cuestiones involucradas con los intere-

ches Bundesamt, "Außenhandel”, Statistisches Jahrbuch, Destatis, Wiesbaden, 2018, pp. 427-428, https://www.destatis.de/DE/Themen/Querschn itt/Jahrbuch/jb-aussenhandel.pdf?_blob=publicationFile\&v=6; H. Kundnani y J. Parello-Plesner, op. cit., p. 2

60 X. Yang, In-depth Discussion: A Look at the Sino-German Relations, Beijing, Institute of European Studies, Chinese Academy of Social Sciences, 2012, http://ies.cass.cn/english/chinare/cer/201212/t20121212_24641 78.shtml

61 Ministerio de Asuntos Exteriores de China, "President Xi Jinping visits the Port of Duisburg, Germany", Duisburgo, 30 de marzo de 2014, http:/ / www.fmprc.gov.cn/mfa_eng/topics_665678/xjpzxcxdsjhaqhfb fwhlfgdgblshlhgjkezzzbomzb_666590/t1164914.shtml

62 K. Zhao, op. cit. 
ses de la UE". ${ }^{63}$ La manera en que se solucionó el contencioso comercial sobre paneles fotovoltaicos entre China y la UE en 2013, en el que los productos chinos se investigaron por dumping, pudo haber contribuido a esta percepción de Beijing (en éste, Alemania prefirió una solución negociada de la UE en lugar de la aplicación de sanciones comerciales que exigían otros miembros como Francia e Italia). ${ }^{64}$

Durante la visita de la canciller Merkel a Beijing, en julio de 2014, el gobierno chino calificó las relaciones bilaterales de "exitosas": en ese momento se oficializaba la nueva agenda de la asociación estratégica. ${ }^{65}$ Sin embargo, el pragmatismo de la "relación especial" puso en evidencia otras limitantes: en octubre de 2014, el primer ministro Li Keqiang realizó una visita oficial a Berlín con la finalidad de llevar a cabo la tercera reunión del Diálogo Intergubernamental y, en contraste, los medios alemanes mostraron más cautela al señalar el delicado momento por el que atravesaba la economía de la UE en ese momento. ${ }^{66}$ Sobre las cuestiones económicas, el gobierno alemán seguía preocupado por temas como la negativa del gobierno chino de abrir sectores de su mercado interno a las empresas alemanas (como las telecomunicaciones y las finanzas), la renuencia a otorgar el Eem a China, la velocidad del aumento de las importaciones de origen chino incluyendo sectores de tecnología avanzada (con lo que China podría presentarse

${ }^{63}$ L. Li, In-depth Discussion: A Look at the Sino-German Relations, Beijing, Institute of European Studies, Chinese Academy of Social Sciences, 2012, http://ies.cass.cn/english/chinare/cer/201212/t20121212_2464178.shtml

${ }^{64}$ C. Goron, "Fighting against Climate Change and for Fair Trade: Finding the EU's Interest in the Solar Panels Dispute with China”, ChinaEU Law Journal, vol. 6, núm. 1-2 (2018), pp. 106-107.

65 Ministerio de Asuntos Exteriores de China, Li Keqiang Meets with Chancellor Angela Merkel of Germany, Beijing, 7 de julio de 2014, https:// www.fmprc.gov.cn/mfa_eng/zxxx_662805/t1172777.shtml; M. Huotari, op. cit., p. 30 .

66 "Economic woes Cloud Germany-China Summit", Deutsche Welle, 9 de octubre de 2014, https:/ / www.dw.com economic-woes-cloud-germanychina-summit/a-17985253/en/ 
como un competidor en el futuro) y el exceso de capacidad de producción de algunos sectores industriales chinos, como es el caso del acero (en julio de 2016, la UE aplicó aranceles compensatorios a las importaciones de acero chino), etc. ${ }^{67}$ Sin embargo, estas tensiones no impidieron que se incrementaran los acuerdos de cooperación científica y tecnológica entre ambos países. ${ }^{68}$

Durante el periodo 2013-2017, el gobierno chino incrementó sus relaciones de cooperación con Berlín conforme avanzaba el proceso de crisis económica y se presentaban otras coyunturas, como la política de pivote estadounidense; en este contexto, como menciona Marcin Przychodniak, China se orientó a fomentar sus relaciones con socios que no la percibieran como una amenaza para sus intereses o el orden internacional: por esta razón, el gobierno chino intensificó los contactos políticos con Alemania en el nivel bilateral (por medio de la asociación estratégica) y multilateral (en mecanismos como las cumbres del G-20) ${ }^{69}$ La política alemana de "revisión", en la que asumía de manera abierta su liderazgo en el sistema internacional, aumentó esta atracción para Beijing, mientras el gobierno alemán continuó con su tendencia a subordinar la agenda de la buena gobernanza (conservando el Diálogo sobre el Estado de derecho como el principal

${ }^{67} \mathrm{Li}$ Wei, op. cit; Gobierno Federal de Alemania, Intensifying cooperation with China, Berlín, 10 de octubre de 2014, https://www.bundesre gierung.de/breg-en/issues/intensifying-cooperation-with-china-413556

68 Ministerio de Asuntos Exteriores de China, Premier Li Keqiang and Chancellor Angela Merkel Discuss China-Germany Innovative Cooperation. Berlín, 11 de octubre de 2014, http://www.fmprc.gov.cn/mfa_eng/topics_66 5678/lkqzlfwdgelsydllhglnzzzbbcxdsjyosnhy/t1200145.shtml

${ }^{69}$ M. Przychodniak, "China's Plans to Strengthen Relations with Germany", Bulletin núm. 65, The Polish Institute of International Affairs, Varsovia, 5 de julio de 2017 , p. 1, https://www.pism.pl/files/?id_plik=23351. La 11 Cumbre del G20 se realizó en Hangzhou en septiembre de 2016, véase Ministerio de Asuntos Exteriores de China, Xi Jinping meets with Chancellor Angela Merkel of Germany, Hangzhou, 5 de septiembre de 2016, http:/ / www.fmprc.gov.cn/mfa_eng/topics_665678/XJPCXBZCESGJTLDRD SYCFHJCXYGHD/t1395342.shtml 
entre los diálogos normativos bilaterales).$^{70}$ En 2016, las relaciones comerciales ascendieron a 170218 millones y por primera vez China se convirtió en el principal socio comercial de Alemania entre los terceros países externos a la UE, sobrepasando a EE.UU. (aunque generando un déficit para Alemania equivalente a 18126 millones).${ }^{71}$ No obstante esta condición deficitaria, poco habitual para la economía alemana, las relaciones comerciales siguieron creciendo: ${ }^{72}$ de manera significativa, durante el respaldo del gobierno de Merkel a las negociaciones de la Asociación Transatlántica de Comercio e Inversiones (TTIP por sus siglas en inglés). ${ }^{73}$

${ }^{70}$ C. Zheng, "Germany's role in EU-China Relations", Chinese Journal of European Studies, vol. 33, núm. 3, Institute of European Studies, Chinese Academy of Social Sciences, Beijing, 2017, http://jtp.cnki.net/bilin gual/detail/html/OZZZ201503001; Cancillería Federal de Alemania, Government Consultations with China: Rule of Law strengthens Trust, Shenyang, 14 de junio de 2016, https://www.bundeskanzlerin.de/Content/ EN/Reiseberi chte/2016/2016-06-10-deutschland-china_en.html Sobre el papel de liderazgo de Alemania, el gobierno de Merkel instauró el concepto de "liderazgo desde el centro". Ver el discurso de la ministra federal de Defensa, Ursula von der Leyen, en la 51 Conferencia de Seguridad de Múnich: U. von der Leyen, Leadership from the Centre, intervención en la $51^{\text {st }}$ Munich Security Conference, Múnich, 6 de febrero de 2015, https://www.securityconference.de/fileadmin/MSC_/2015/Freitag/150 206-2015_Rede_vdL_MSC_Englisch-1_Kopie_.pdf

${ }^{71}$ Statistisches Bundesamt, "Außenhandel", op. cit., pp. 427-428.

${ }^{72}$ Rene Wagner y Michael Nienaber, "China steams past U.S., France to be Germany's biggest Trading Partner", Reuters, 23 de febrero de 2017, https://www.reuters.com/article/us-germany-economy-trade-idUS KBN1622SO

${ }^{73}$ Cancillería Federal de Alemania, Angela Merkel for the Free Trade Agreement: "TTIP is a fantastic opportunity", Berlín, 21 de septiembre de 2015, https://www.bundeskanzlerin.de/bkin-en/news/-ttip-is-a-fantasticopportunity-430262 
La agenda de la asociación estratégica integral sino-alemana de 2014

En la investigación se ha considerado como asociación estratégica a un vínculo organizacional establecido en la relación bilateral entre dos actores internacionales (por lo general países y organizaciones internacionales). En 1993, China hizo oficial su primer acuerdo de asociación estratégica con Brasil; desde entonces, Beijing ha buscado establecer acuerdos de asociación con otros actores internacionales con la finalidad de contar con una mayor cantidad de socios que pudieran contribuir a consolidar el proceso de modernización chino (especialmente en las áreas de cooperación económica). Las asociaciones también son instrumentos diplomáticos con los que China ha diversificado sus relaciones con el exterior: en la actualidad, Beijing mantiene asociaciones estratégicas con más de sesenta socios. ${ }^{74}$

En contraste, durante la década de 1990, los gobiernos de Alemania prefirieron restringir los vínculos denominados "asociaciones estratégicas" a sus aliados más cercanos, como EE.UU. y sus socios en el entorno europeo: las "relaciones especiales de Alemania" consistían tanto de intereses materiales, como de "una historia compartida, valores e ideas".

Como lo menciona Felix Heiduk, inicialmente los vínculos de asociación estratégica de Alemania se orientaron por criterios como: "Los lazos históricos de larga duración, los intereses compartidos en una variedad de áreas políticas y un sentido común de proyectos y objetivos basados en ideas y valores compartidos". ${ }^{75}$ Sin embargo, esta situación comenzó a cambiar a partir de algunas coyunturas internacionales concretas durante la década del 2000, como la mayor pre-

${ }^{74}$ F. J. Haro Navejas y R. Hernández Mendoza, "Viaje al oeste. La Asociación sino-mexicana: una posibilidad funcionalista”, Foro Internacional, vol. 56, núm. 2 (2016), pp. 422-424.

${ }^{75}$ F. Heiduk, "What is in a name? Germany's Strategic Partnerships with Asia’s Rising Powers”, Asia Europe Journal, vol. 13, núm. 2 (2015), p. 132. 
sencia de China en la economía mundial y el periodo de distanciamiento transatlántico. ${ }^{76}$

A partir de 2000 y durante esa década, el gobierno alemán estableció acuerdos de asociación estratégica con las denominadas "potencias emergentes", como China e India, para así impulsar un proceso de institucionalización de sus relaciones bilaterales. ${ }^{77}$ En la actualidad Alemania mantiene vínculos de asociación con ocho países. ${ }^{78}$

Por su parte, la agenda de la asociación estratégica integral sino-alemana se compone de alrededor de setenta diálogos sectoriales (en 2014 eran sesenta), además de la cumbre de alto nivel y el diálogo intergubernamental (este mecanismo realizó su quinta reunión en 2018) ${ }^{79}$ Sumados a los diálogos sectoriales, la asociación sino-alemana se compone por un conjunto de diálogos semioficiales y "estructuras de diálogo". Los tres pilares de éstos son: 1) los diálogos políticos (que incluye aquéllos sobre el fortalecimiento del Estado de derecho en China y el diálogo sobre derechos humanos); 2) los diálogos sobre cooperación económica y negocios; 3) los diálogos sobre contactos pueblo a pueblo, sociedad civil y cooperación científica y tecnológica (como sobre medio ambiente, cambio climático, energías renovables y control de contaminantes).$^{80}$

${ }^{76}$ H. Kundnani y J. Parello-Plesner, op. cit., p. 6

${ }^{77}$ F. Heiduk, 2015, art. cit., p. 131.

78 A. Narlikar y J. Plagemann, "Making the most of Germany's Strategic Partnerships: A Five-Point Proposal”, Giga Focus Global núm 6, Hamburgo, German Institute of Global and Area Studies (2016), p. 2, https:/ / www.giga-hamburg.de/en/system/files/publications/gf_global_1606. pdf Los socios estratégicos de Alemania son: Australia, Brasil, China, Emiratos Árabes Unidos, India, Indonesia, Vietnam y Sudáfrica.

${ }^{79}$ Gobierno Federal de Alemania, Joint Declaration between Germany and China: Comprehensive Strategic Partnership, 2014, op. cit.; Ministerio Federal de Asuntos Exteriores de Alemania, Joint Declaration from the fifth German-Chinese Intergovernmental Consultations "Responsible Partners for a better World", Berlín, 9 de julio de 2018, https:/ / www.auswaertiges-amt.de/bl ob/2123764/3c77182c7e36afe693521a8a68ad0af7/joint-declarationfrom-the-5th-german-chinese-intergovernmental-consultations-data.pdf

${ }^{80}$ M. Huotari, op. cit., pp. 31-32. 
También existen diálogos de consulta entre los Ministerios de Defensa y de Asuntos Exteriores. Desde 2013 se estableció un Diálogo estratégico y de asuntos globales, desde el cual se han abordado cuestiones de coyuntura internacional, como el conflicto en Ucrania y Crimea (desde 2014), la seguridad en Asia del Este, la cuestión nuclear iraní, etc. Los diálogos sectoriales sobre cooperación en negocios, tecnología e innovación son prioritarios en la agenda de la asociación: ${ }^{81}$ en 2014 se constituyó un Diálogo consultivo sobre negocios (enfocado en temas como acceso a mercados, cooperación en innovación, la internacionalización del yuan renminbi y la expansión de la presencia del empresariado alemán en China), se acordó un Plan de Acción sobre Cooperación Conjunta en Innovación y, en 2015, se establecieron un Diálogo de alto nivel sobre finanzas, así como un Diálogo sobre seguridad informática. ${ }^{82}$ Las visitas realizadas a China por la canciller y los ministros del gobierno alemán suelen estar integradas por delegaciones de federaciones empresariales como el Comité de Empresarios Alemanes para Asia Pacífico, la Asociación Bancaria y la Cámara de Comercio Alemana y Europea quienes representan los intereses alemanes (y europeos) ante las instituciones $\mathrm{y}$ funcionarios de alto nivel del gobierno chino y el PCCh. ${ }^{83}$ De manera significativa, la cooperación sino-alemana considera que su asociación "[...] es una parte importante de la asociación estratégica integral China-UE: ambas se refuerzan mutuamente". ${ }^{84}$

${ }^{81}$ Ministerio de Asuntos Exteriores de China, Li Keqiang and Chancellor Angela Merkel of Germany Co-chair the 4th Round of China-Germany InterGovernmental Consultation, Beijing, 13 de junio de 2016, https://www. fmprc.gov.cn/mfa_eng/zxxx_662805/t1372371.shtml

${ }^{82}$ M. Huotari, op. cit., p. 32.

${ }^{83}$ Loc. cit

${ }^{84}$ Gobierno Federal de Alemania, Joint Declaration between Germany and China: Comprehensive Strategic Partnership, 2014, op. cit. 


\section{EL CUARTO PERIODO (2017): LA RELACIÓN SINO-ALEMANA DURANTE LA PRESIDENCIA DE DONALD TRUMP}

Durante los cuatro gobiernos de Angela Merkel se ha mantenido de forma estable una prioridad estructural de la política exterior de Alemania: la solidez de sus compromisos con los mecanismos de cooperación económica, política y de defensa, pertenecientes a la relación transatlántica (especialmente en el caso de la oTAN). Sin embargo, a partir de 2017, con el inicio del periodo de gobierno de Donald Trump en EE.UU., la relación germano-estadounidense ha sufrido cierto distanciamiento diplomático. No obstante la coyuntura actual, el gobierno de Merkel (en conjunto con sus ministros de Exteriores y Comercio) ha mostrado una clara mesura ante la incontinencia verbal de Trump y sus decisiones políticas, como el abandono de las negociaciones del TTIP, el apoyo al Brexit y las críticas al gobierno alemán: ello ha sido una clara muestra del compromiso con la estabilidad de la relación transatlántica.

En lo correspondiente a la relación sino-alemana, si bien las declaraciones de Trump han contribuido a la formación de consensos en los diálogos que mantienen ambos países, igualmente los actores han sido cuidadosos de no emitir declaraciones o críticas abiertas en contra de las posturas del gobierno estadounidense: es decir, China y Alemania han evitado asumir posiciones que puedan interpretarse como políticas de contención en contra de EE.UU. ${ }^{85}$ Los consensos en la relación sino-alemana, formados en 2017-2018, se relacionan con la defensa del sistema de libre comercio, el apoyo al proceso de integración europeo, la defensa del Acuerdo sobre el Cambio Climático de París, las críticas al proteccionismo y la defensa del acuerdo nuclear con Irán, tras haber

${ }^{85}$ Cancillería Federal de Alemania, Taking Good Relations to a New Level. Germany and China intend to further expand their Relations in many Areas, stressed Chancellor, Berlin, 5 de julio de 2017, https://www.bundeskanzle rin.de/Content/EN/Artikel/2017/07_en/2017-07-04-staatsbesuch-xijinping_en.html 
sido desconocido por Trump. De manera coincidente, la visita oficial de Merkel a Beijing, en mayo de 2018, ocurrió al mismo tiempo que el gobierno de Trump impusiera sanciones arancelarias contra China, Canadá, México y la ue (Merkel y Li Keqiang se reunirían en julio, en ocasión de la reunión del Dialogo intergubernamental). ${ }^{86}$

Posteriormente, en diciembre de 2018, durante la celebración de la $13^{\mathrm{a}}$ Cumbre del G-20, en Buenos Aires, Merkel y Xi Jinping sostuvieron su segunda reunión del año, en la que evaluaron la mayor apertura del mercado chino a las empresas alemanas y reivindicaron "salvaguardar el multilateralismo y una economía mundial abierta". ${ }^{87}$ Como muestra de las políticas proteccionistas del gobierno de Trump, puede mencionarse la prohibición de EE.uU., de mayo de 2019, del uso de equipos de tecnología digital 5G de compañías chinas, como Huawei, por compañías estadounidenses en su territorio (argumentando motivos de "seguridad nacional"). En contraste, Alemania ha resistido (en una posición compartida por la mayoría de los miembros de la UE) a las presiones estadounidenses para implementar una prohibición generalizada, entre los aliados occidentales, al uso de la tecnología 5G de China. Hasta el momento, el gobierno alemán ha anunciado que continuará con los contratos con compañías chinas para la implementación de la red 5G en su territorio, aunque sería preferible establecer un acuerdo de "no espionaje" con Beijing para proseguir con la "construcción de una red de telefonía de nueva generación". ${ }^{88}$ Asimismo, el gobierno alemán exige a las compañías de capital chino un

${ }^{86}$ Cancillería Federal de Alemania, Angela Merkel in China: Cooperating Well and Fairly, Beijing, 25 de mayo de 2018, https://www.bundeskan zlerin.de/bkin-en/news/cooperating-well-and-fairly-1143250

87 "Xi pledges joint Efforts with Germany to safeguard Multilateralism, Open Economy", People's Daily, Buenos Aires, 1 de diciembre de 2018, http:/ / en.people.cn/n3/2018/1202/c90000-9524363.html

88 "A 'No-spy' Deal would help any Huawei bid - Germany", Reuters, 14 de mayo de 2019, https://www.reuters.com/article/china-germany/a-no -spy-deal-would-help-any-huawei-bid-germany-idUSS8N21G011 
respeto estricto de las leyes alemanas y de la UE durante sus actividades comerciales y de inversión en su territorio.

\section{Consideración FinAL}

En la investigación se analizó el desarrollo de la relación bilateral entre China y Alemania durante el periodo 2005-2018: dos potencias económicas que se caracterizan por sus asimetrías pero que han elaborado un esfuerzo diplomático para construir mecanismos de diálogo y cooperación que contribuyan a profundizar sus relaciones de cooperación económica. En este proceso han resultado fundamentales dos elementos estructurales: por un lado, la ausencia de una rivalidad geopolítica entre ambos países debido a la distancia geográfica que los separa; por otro, la necesidad de las dos principales potencias económicas en Europa y Asia de mantener relaciones comerciales estables a partir del periodo de la crisis económica mundial iniciado en 2008.

Durante la crisis, la relación sino-alemana tuvo como prioridad la profundización de su interdependencia, algo que se reflejó en la evolución ascendente de sus transacciones comerciales, en lugar de que ocurriera un escenario de ruptura entre ambos países. Por el contrario, la relación bilateral sino-alemana profundizó su proceso de institucionalización mediante la construcción de nuevos mecanismos de diálogo y cooperación, con la asociación estratégica integral que ambos actores redefinieron entre 2010 y 2014; sin embargo, el gobierno alemán debió subordinar la promoción de una agenda normativa con el objetivo de mantener la estabilidad. Esto significa que el gobierno alemán respetó los principios cardinales de la política exterior china, consistentes en la salvaguarda de su soberanía y la no intervención.

De la misma manera, en la investigación se identificó que la reducción de una agenda normativa por Alemania no incluyó algunos principios estructurales de su política exterior que se remontan al periodo de Guerra Fría: es decir, los com- 
promisos del gobierno alemán con el mantenimiento de la Alianza Atlántica y la preservación de un orden global, basado en el derecho internacional y el sistema económico liberal. En este proceso de acomodo de intereses, debe resaltarse que la redefinición de la asociación estratégica sino-alemana se oficializó durante el contexto del debate sobre la Revisión 2014 del ministro Steinmeier. Sin embargo, hasta el momento parece existir un consenso entre ambos gobiernos sobre el respeto de sus respectivos valores normativos (algo reafirmado por la presencia de un discurso antieuropeísta y proteccionista como el de Donald Trump): en un momento de incertidumbre en la relación transatlántica, la reafirmación de los compromisos de Alemania con sus aliados occidentales contribuye a delimitar con mayor claridad los términos de la relación sino-alemana (y evitar disonancias cognitivas). Hasta el momento, las relaciones de interdependencia entre China y Alemania no han impulsado un proceso de cambio estructural en el sistema internacional en cuanto a un cambio de las relaciones estratégicas tradicionales en la relación transatlántica, sin embargo, estos principios no impiden el desarrollo de la cooperación pragmática bilateral y cambios en la estructura del comercio global (a partir de 2016, China sobrepasó a EE.UU. como el principal socio comercial de Alemania, externo a la UE).

En la actualidad, la relación bilateral continúa con la profundización de sus relaciones de interdependencia económica aunque enfrenta algunos retos: 1) las consecuencias a largo plazo de la política exterior antieuropeísta ejercida por Trump (y la orientación respecto de un nuevo gobierno alemán que sustituya a Angela Merkel), así como la posición que podrían ocupar países como China, India y Rusia para la política exterior alemana en el aspecto estructural o bien, sólo coyuntural; 2) el fortalecimiento del sector exportador chino que podría presentarse, de manera creciente, como un rival comercial para la industria alemana de alto valor agregado (considerando el déficit comercial); 3) la creciente presencia económica de China en el espacio europeo, par- 
ticularmente en el incremento de las inversiones de origen chino en el sector industrial alemán; 4) de manera similar, el aumento de los intereses de China en Europa del Este, mediante las inversiones de empresas de capital chino y la instrumentación de los proyectos de la Iniciativa Cinturón y Ruta de la Seda. La negociación de estos temas demostrará la solidez de los mecanismos de diálogo y cooperación que forman parte de la agenda de la asociación estratégica sinoalemana.

\section{Bibliografía}

“A 'No-spy' Deal would help any Huawei bid - Germany”, Reuters, 14 de mayo de 2019, https://www.reuters.com/article/china-ger many/a-no-spy-deal-would-help-any-huawei-bid-germanyidUSS8N21G011

Bendiek, Annegret, “The '2014 Review': Understanding the Pillars of German Foreign Policy and the Expectations of the rest of the World", Working Paper RD EU/Europe núm 2015/05, Berlín, Stiftung Wissenschaft und Politik, 2015.

BräUner, Oliver, "Beyond the Arms Embargo: EU Transfers of Defense and Dual-Use Technologies to China", Journal of East Asian Studies, vol. 13, núm. 1, 2013.

Cancillería Federal de Alemania, Angela Merkel in China: Cooperating Well and Fairly, Beijing, 25 de mayo de 2018, https://www. bundeskanzlerin.de/bkin-en/news/cooperating-well-andfairly-1143250

Cancillería Federal de Alemania, Taking Good Relations to a New Level. Germany and China intend to further expand their Relations in Many Areas, stressed Chancellor, Berlin, 5 de julio de 2017, https://www. bundeskanzlerin.de/Content/EN/Artikel/ 2017/07_en/201707-04-staatsbesuch-xi-jinping_en.html

Cancillería Federal de Alemania, Government Consultations with China: Rule of Law strengthens Trust, Shenyang, 14 de junio de 2016, https://www.bundeskanzlerin.de/Content/EN/Reiseberi chte/2016/2016-06-10-deutschland-china_en.html 
Cancillería Federal de Alemania, Angela Merkel for the Free Trade Agreement: "TTIP is a fantastic opportunity", Berlín, 21 de septiembre de 2015, https://www.bundeskanzlerin.de/bkin-en/ news/-ttip-is-a-fantastic-opportunity-430262

Cabestan, Jean-Pierre, "La politique étrangère chinoise: une Chine sans ennemis n'est pas forcément une Chine rassurante", Hérodote, núm. 125, 2007.

Casarini, Nicola, "The Evolution of the EU-China Relationship: from Constructive Engagement to Strategic Partnership", Ocassional Paper, núm. 64, París, Euiss, 2006.

"China cancels Human Rights Meeting after Dalai Lama Row", Deutsche Welle, 14 de octubre de 2007, https://www.dw.com/ en/china-cancels-human-rights-meeting-after-dalai-lamarow/a-2824545

"China, Germany to hold Annual Consultations to deepen 'Strategic Partnership', Deutsche Welle, 16 de julio de 2010, http:// www.dw.de/china-germany-to-hold-annual-consultations-todeepen-strategicpartnership/a-5809459

Connelly, Marisela, "Comercio y consumo de opio en China”, Estudios de Asia y África, vol. 25, núm. 3 (83), 1990.

Consejo Europeo, $11^{\text {th }}$ EU-China Summit Prague Joint Press Communiqué 10234/09 (Presse 147), Bruselas, 20 de mayo de 2009, https:/ / ec.europa.eu/clima/sites/clima/files/docs/0023/ joint_statement_en.pdf

Crotty, William, "Armageddon, just another Campaign or something in between. The Meaning and Consequences of the 2004 Presidential Election", Crotty, William (ed.) A Defining Moment: The Presidential Election of 2004, Nueva York, Routledge, 2015.

Dirección General de Comercio de la Comisión Europea, European Union, Trade in goods with China, Bruselas, 19 de marzo de 2019, https://webgate.ec.europa.eu/isdb_results/factsheets/country/details_china_en.pdf

"Economic woes Cloud Germany-China Summit", Deutsche Welle, 9 de octubre de 2014, https:/ / www.dw.com/en/economic-woescloud-germany-china-summit/a-17985253 
Forsberg, Tuomas, "From Ostpolitik to "Frostpolitik"? Merkel, Putin and German Foreign Policy towards Russia", International Affairs, vol. 92, núm. 1, 2016.

GAUck, Joachim, “Germany's role in the world: Reflections on Responsibility, Norms and Alliances" (Discurso de apertura de la $50^{\text {th }}$ Munich Security Conference), Múnich, 31 de enero de 2014, http://www.bundespraesident.de/SharedDocs/Reden/EN/ JoachimGauck/Reden/2014/140131-Munich-Security-Con ference.html

Gajauskaité, Ieva, "Strategic Partnerships in Foreign Policy: Comparative Analysis of Polish -Ukrainian and Lithuanian - Ukrainian Strategic Partnerships", Lithuanian Annual Strategic Review, vol. 11, núm. 1, 2013.

Gobierno Federal de Alemania, Intensifying cooperation with China, Berlín, 10 de octubre de 2014, https:/ / www.bundesregierung. de/breg-en/issues/intensifying-cooperation-with-china-413556

Gobierno Federal de Alemania, Joint Declaration between Germany and China: Comprehensive Strategic Partnership, Berlín, The Press and Information Office of the Federal Government, 28 de marzo de 2014, https://www.bundesregierung.de/Content/EN/Pressemitteilungen/BPA/2014/2014-03-28-china-declaration.html

Godement, François, "Key issues of China's Leadership Succession and their Relevance to the EU", en Casarini, Nicola (ed.), Brussels-Beijing: changing the game?, Report núm. 14, París, Euiss, 2013.

Goron, Coraline, "Fighting against Climate Change and for Fair Trade: Finding the EU's Interest in the Solar Panels Dispute with China”, China-EU Law Journal, vol. 6, núm. 1-2, 2018.

GotTwald, Joern-Carsten, "Germany's China-Policy: Trade Promotion, Human Rights and European Disunity", en Marco Overhaus et. al. (eds.), German-Chinese Relations: Trade Promotion Plus Something Else? German Foreign Policy in Dialogue, vol. 6, núm. 16, 2005.

Grosse, Tomasz, "Geoeconomic Relations Between the EU and China: The Lessons from the EU Weapon Embargo and from Galileo", Geopolitics, vol. 19, núm.1, 2014. 
Haro Navejas, Francisco Javier y Rosángel Hernández MendozA, "Viaje al oeste. La Asociación Sino-mexicana: una posibilidad funcionalista", Foro Internacional, vol. 56, núm. 2, 2016.

Heiduk, Felix, "What is in a name? Germany's Strategic Partnerships with Asia's Rising Powers", Asia Europe Journal, vol. 13, núm. 2, 2015.

Heiduk, Felix, "Conflicting Images? Germany and the Rise of China”, German Politics, vol. 23, núm. 1-2, 2014.

Huotari, Mikko, "Germany's China Policy: No Honeymoon Forever" en Huotari, Mikko et. al. (eds.), Mapping Europe-China Relations. A Bottom-Up Approach, Berlín, MERICs, 2015.

Keohane, Robert O. y Joseph S. Nye, "La interdependencia en la política mundial”, en Arturo Borja (comp.), Interdependencia, Cooperación y Globalismo. Ensayos escogidos de Robert O. Keohane, México, CIDE, 2005.

Kundnani, Hans, "Leaving the West. Germany looks East", Foreign Affairs, enero-febrero de 2015.

Kundnani, Hans y Jonas Parello-Plesner, "China and Germany: Why the Emerging Special Relationship matters for Europe”, Policy Brief, núm. 55, Londres, ECFr, 2012.

Langenbacher, Eric y Ruth Wittlinger, "The End of Memory? German-American Relations under Donald Trump", German Politics, vol. 27, núm. 2, 2018.

LEYEN, Ursula von der, Leadership from the Centre (intervención en la $51^{\text {st }}$ Munich Security Conference), Múnich, 6 de febrero de 2015, https://www.securityconference.de/fileadmin/MSC_/ 2015/Freitag/150206-2015_Rede_vdL_MSC_Englisch-1_Ko pie_.pdf

Li, Lezeng, In-depth Discussion: A Look at the Sino-German Relations, Beijing, Institute of European Studies, Chinese Academy of Social Sciences, 2012, http://ies.cass.cn/english/chinare/ cer/201212/t20121212_2464178.shtml

LI, Wei, The Limits of the China-Germany Special Relationship, Beijing, Institute of European Studies, Chinese Academy of Social Sciences, 2014, http:/ /ies.cass.cn/wz/mtcf/201404/t20140409 _2463171.shtml 
"Merkel pushes China to open its Markets", The Local, 16 de julio de 2010, https://www.thelocal.de/20100716/28545

Miller, Bernhard y Wolfgang C. Müller, "Managing Grand Coalitions: Germany 2005-09”, German Politics, vol. 19, núm. 3-4, 2010.

Ministerio Federal de Asuntos Exteriores de Alemania, Joint Declaration from the fifth German-Chinese Intergovernmental Consultations "Responsible Partners for a better World", Berlín, 9 de julio de 2018, https://www.auswaertiges-amt.de/blob/2123764/3c771 82c7e36afe693521a8a68ad0af7/joint-declaration-from-the5th-german-chinese-intergovernmental-consultations-data.pdf Ministerio de Asuntos Exteriores de China, Xi Jinping meets with Chancellor Angela Merkel of Germany, Hangzhou, 5 de septiembre de 2016, http://www.fmprc.gov.cn/mfa_eng/topics_665678/ XJPCXBZCESGJTLDRDSYCFHJCXYGHD/t1395342.shtml

Ministerio de Asuntos Exteriores de China, Li Keqiang and Chancellor Angela Merkel of Germany Co-chair the 4th Round of ChinaGermany Inter-governmental Consultation, Beijing, 13 de junio de 2016, https://www.fmprc.gov.cn/mfa_eng/zxxx_662805/ t1372371.shtml

Ministerio de Asuntos Exteriores de China, Li Keqiang Meets with Chancellor Angela Merkel of Germany, Beijing, 7 de julio de 2014, https://www.fmprc.gov.cn/mfa_eng/zxxx_662805/t1172777. shtml

Ministerio de Asuntos Exteriores de China, Premier Li Keqiang and Chancellor Angela Merkel Discuss China-Germany Innovative Cooperation, Berlín, 11 de octubre de 2014, http://www.fmprc.gov. cn/mfa_eng/topics_665678/lkqzlfwdgelsydllhglnzzzbbcxdsj yosnhy/t1200145.shtml

Ministerio de Asuntos Exteriores de China, President Xi Jinping Visits the Port of Duisburg, Germany, Duisburgo, 30 de marzo de 2014, http://www.fmprc.gov.cn/mfa_eng/topics_665678/xjp zxcxdsjhaqhfbfwhlfgdgblshlhgjkezzzbomzb_666590/t11 64914.shtml

Ministerio de Asuntos Exteriores de China, Address by H.E. Wen Jiabao Premier of the State Council of the People's Republic of China at the China-Germany Business Summit, Hannover, 23 de abril de 
2012, http://www.fmprc.gov.cn/mfa_eng/topics_665678/wjb ispg_665714/t930826.shtml

Ministerio de Asuntos Exteriores de China, Hu Jintao Meets with German Chancellor Merkel. Beijing, 16 de julio de 2010, http:/ / www.china-embassy.org/eng/zgyw/t717842.htm

Ministerio de Asuntos Exteriores de China, Joint Statement of the 12th China-EU Summit, Nanjing, 30 de noviembre de 2009, http:/ / www.chinamission.be/eng/zywj/zywd/t1228338.htm

Naughton, Barry, "The Challenges of Economic Growth and Reform”, en Robert S. Ross y Jo Inge Bekkevold (eds.), China in the Era of Xi Jinping: Domestic and Foreign Policy Challenges, Washington, Georgetown University Press, 2016.

Przychodniak, Marcin, "China's Plans to Strengthen Relations with Germany”, Bulletin núm. 65, Varsovia, The Polish Institute of International Affairs, 5 de julio de 2017, https:/ /www.pism. $\mathrm{pl} /$ files/?id_plik=23351

Rupprecht, Klaus, "Germany's Policy toward China and the SARs of Hong Kong and Macau”, en Miguel Santos-Neves y Brian Bridges, (ed.), Europe, China and the two Sars: towards a New Era, Londres, Macmillan Press, 2000.

SAndschneider, Eberhard, "China's Diplomatic Relations with the States of Europe”, China Quarterly, núm. 169, 2002.

Schnellbach, Christoph y Joyce Man, "Germany and China: ¿Embracing a Different Kind of Partnership?”, CAP Working Paper, Múnich, CAP, University of Munich, 2015.

"Schröder calls for end to Arms Embargo against China", Deutsche Welle, Beijing, 3 de diciembre de 2003, https:/ /www.dw.com/ en/schr\%C3\%B6der-calls-for-end-to-arms-embargo-againstchina/a-1047143

SteInmeIER, Frank-Walter, Speech by Foreign Minister Frank Walter Steinmeier at the $50^{\text {th }}$ Munich Security Conference, Múnich, Ministerio Federal de Asuntos Exteriores de Alemania, 31 de enero de 2014, https://www.auswaertiges-amt.de/en/newsroom/news/ 140201-bm-muesiko/259556

SteinmeIer, Frank-Walter, Speech by Dr Frank-Walter Steinmeier, Federal Minister for Foreign Affairs, at the Handover Ceremony on 17 December 2013, Berlín, Ministerio Federal de Asuntos Exterio- 
res de Alemania, 17 de diciembre de 2013, https://www. auswaertiges-amt.de/en/newsroom/news/131217-bm-an trittsrede/258766

Statistisches Bundesamt, China is Germany's main Trading Partner once again, Destatis, Wiesbaden, 18 de febrero de 2019, https:/ / www.destatis.de/EN/Press/2019/02/PE19_057_51.html

Statistisches Bundesamt, "Außenhandel”, Statistisches Jahrbuch, Destatis, Wiesbaden, 2018, https://www.destatis.de/DE/Themen/ Querschnitt/Jahrbuch/jb-aussenhandel.pdf?_blob=publi cationFile\&v $=6$

Talley, Ian y Jeffrey Sparshott, "U.S. Blasts Germany's Economic Policies", The Wall Street Journal, 31 de octubre de 2013, https://www.wsj.com/articles/us-treasury-targets-german-economic-policies-1383170134

Terhalle, Maximiliam y Giegerich Bastian, "The Purpose of the German Power", en James Bindenagel, Mathias Herdegen y Karl Kaiser (eds.), International Security in the $21^{\text {st }}$ Century. Germany's International Responsibilities, Bonn, University Press, 2017.

VÉdrine, Hubert, "L'Émergence de la Chine vue de France", Politique Étrangère, núm. 3, 2006.

WAgner, Rene, "Exclusive: German Trade Surplus with U.S. declined in 2018 but was still almost 49 Billion Euros", Reuters, 11 de febrero de 2019, https: / /www.reuters.com/article/us-germanyeconomy-exclusive/exclusive-german-trade-surplus-with-u-s-de clined-in-2018-but-was-still-almost-49-billion-euros-idUSKCN 1Q01EY

Wagner, Rene y Michael Nienaber, "China steams past U.S., France to be Germany's biggest Trading Partner”, Reuters, 23 de febrero de 2017, https://www.reuters.com/article/us-ger many-economy-trade-idUSKBN1622SO

WANG, Jisi, " 'Marching Westwards': The Rebalancing of China's Geostrategy", en Shao Binhong (ed.), The World in 2020 According to China: Chinese Foreign Policy Elites discuss Emerging Trends in International Politics, Leiden, Brill, 2014.

Wu, Po-Kuan y Mads Dagnis Jensen, "Examining the EU-China Relationship in the Aftermath of the Economic Crisis", International Journal of Public Administration, vol. 40, núm. 14, 2017. 
"Xi pledges joint Efforts with Germany to safeguard Multilateralism, Open Economy", People's Daily, Buenos Aires, 1 de diciembre de 2018, http://en.people.cn/n3/2018/1202/c9000 0-9524363.html

YANG, Xiepu, In-depth Discussion: A Look at the Sino-German Relations, Beijing, Institute of European Studies, Chinese Academy of Social Sciences, 2012, http:/ /ies.cass.cn/english/chinare/cer/20 1212/t20121212_2464178.shtml

Zhang, Xiaotong, EU-China Economic Diplomacy: When Economics meets Politics, Wuhan, Wuhan University Research Centre for Economic Diplomacy, Wuhan University, 2014, http://ced.whu.edu. $\mathrm{cn} /$ Show/?id=103\&siteid=3

ZhaO, Ke, "Germany's Diplomatic Rebalancing and Its Impact on China”, China International Studies, núm. 66, 2017, https:// www.pressreader.com/china/china-international-studies-engli sh/20170920/281500751430764

Zhao, Ke, "Does Ascendant Germany pose a Challenge for China?", Chinese Social Sciences Today, Beijing, 3 de mayo de 2016, http://english.cssn.cn/research/internationalstudies/2016 03/t20160308_2903111.shtml

Zheng, Churning, "Germany's role in EU-China Relations", Chinese Journal of European Studies, vol. 33, núm. 3, Beijing, Institute of European Studies, Chinese Academy of Social Sciences, 2017, http://jtp.cnki.net/bilingual/detail/html/OZZZ20150 3001 